\title{
Writing a scientific paper: an integral part of the continuous academic development of healthcare professionals
}

"Continuing medical education", "lifelong learning", "continuous professional development", "physicians as scholars" - several terms with ovelapping meaning and with a common aim. They stress the ethical obligation of healthcare professional to ensure the high quality of health care and its continuous improvement, by continuous learning, by keeping healthcare professional at the latest level of knowledge in the field $(1,2)$. Lifelong learning and continuous medical education has become the natural part of medicine.

The continuous education has two fundamental complimentary aspects: learning and teaching/ sharing experience. The first one - the learning - is more stresses and understood, and the concepts, methods and content are continuously optimized. Slightly different situation is with teaching/ sharing experience. The healthcare professionals have also a natural obligation to share their experience, to mentor and to train junior colleagues. It requires skills and knowledge to teach the other, to share the knowledge and experience, including the identification of gaps in knowledge, resources to correct those deficiencies, overall assessment of knowledge, feedback about communication skills and professionalism, and methods to evaluate and improve one's practice (3).

The healthcare professionals are not prepared fully for these responsibilities.It is assumed that sharing experience and mentoring are something natural to educated people. However, it requires specific skills. And besides the natural counseling by senior teachers in everyday practice - the basis for learning others and sharing an experience is writing scientific papers. It is stressed that writing a scientific paper should be a natural part of the professional development (4-7). Even if you are not a researcher, your professional experience is valuable and worth to be shared.

This aspect is not always sufficiently recognized by healthcare professionals as a required part of medical education, including the continuous medical education. Traditionally, the focus of CME is dominantly on the discipline specific subjects - on the particular medical field. The aspect of research and sharing experience/ teaching/ mentoring is not a part of curricula.

Writing research/ scientific papers is one of methods for teaching others as well as for your own learning. Additionally to your personal development it brings you national and international recognition, prepares you for collaboration, and increases your competitiveness. The systematic approach to writing helps you not only to publishing in internationally recognized scientific journals, but also to active and qualified participation at international conferences, and projects.

Writing research/ scientific papers has well defined rules, it is learnable and number of universities teaches systematically students "how to write“. Basically, the process of writing a research/ scientific paper consists of four major interrelated components:

- Definition of a research problem: how to turn the idea/ recognized problem into a strong clear message;

- Research study protocol preparation: how to do research;

- Academic writing skills: how to write a manuscript;

- English and Academic English: how to provide clear and consistent message using the proper language.

\section{The idea and definition of a problem:}

At the beginning there is the identification of a research problem, this step requires critical appraisal skills. The interest can arise from a need to summarize and categorise the current level of knowledge or from identified gaps in knowledge and controversies in evidence.

\section{Research study protocol preparation}

For a concrete research study, it is then vital to prepare a detailed consistent study protocol. Additionally to the descriptive parts, such as the „material and methods" part, it has to include the objectives of the study, clearly defined aim, 
reasoning for the significance of the study and a brief overview of current level of knowledge. Written in the proper way, it prepares basis for the publication that should be an natural accomplishment of every research study.

This emerging Journal is related to the initiative of the International Research Interdisciplinary School (IRIS) that provides an interdisciplinary international environment for training skills required for preparing research study protocols, scientific manuscript and international collaboration (8-10). The IRIS goals are to:

- Develop skills to carry out research projects, based on a practical, problembased approach,

- Increase communication skills, including the skills in argumentation, negotiation and critical appraisal,

- Develop skills in international research team building and networking, and establish enjoyable cross discipline/cross cultural collaboration,

- Encourage researchers to publish scientific papers.

The evaluation of the scientific performance of trainees for the period $2007-2013$ showed that the IRIS training was associated with identifiable increase in scientific publication activity (9)

\section{Academic writing skills}

Academic writing skills refer to skills related to writing a concrete manuscript of a research paper. The structure and content of a research paper follow specific rules and requirements, and it is recommended to know and understand them. Also, it is important to realize that the process of writing needs to be addressed systematically, and it comprises of several steps, starting with a good planning and continuing with systematic writing. An efficient way of writing a manuscript is to start writing parallel with the preparation of the study protocol. Another important skill is handling comments is an important skill of peers, co-authors, coworkers, and later of journal reviewers. It is necessary to realize that rewriting of the manuscript is an integral part of the process and definitely not a sign of failure. And finally, the motivation for writing is essential - it is a tool for achieving a particular objective.

Not all universities include academic writing skills into their curricula, but there is number of information on the Internet, either courses or books, some of them are listed below.

\section{English and Academic English}

English language is nowadays the language that dominates in the international scientific environment, what is a strong motivation to master this language at acceptable level also by non-native English speakers. Academic English differs from the "spoken" or "literature" language. The difference is not only in using specific medical terminology, but there are also specific rules and recommendations. For example, also using tenses in academic writing differs from "spoken" English and has specific meaning.

In this Journal we want to encourage and motivate you - our readers and colleagues, including physicians-in-training or practicing physicians, to contribute by your knowledge and personal experience to the global knowledge. We strongly support the idea of incorporating the writing of scientific papers at the medical training and portfolio of continuous education and professional development, as essential professional skills throughtout medical training and practice.

Several recommended links:

http://www.helsinki.fi/kksc/language.services/Aca dWrit.pdf

http://2012books.lardbucket.org/pdfs/successfulwriting.pdf

http://www.uefap.com/materials/history/eas_ss.pd $\underline{f}$

https://www.tcd.ie/CAPSL/readysteadywrite/Acad emicWritingHandbook.pdf https://americanenglish.state.gov/files/ae/resource files/developing_writing_1.pdf http://media.wix.com/ugd/cf6032_175a8ffed5ae41 40b7f0e3a946581d37.pdf https://www.youtube.com/watch?v=mZQgd2sPxp k 
Ljuba Bacharova ${ }^{1,2}$

${ }^{1}$ International Laser Center, Bratislava, Slovak Republic

${ }^{2}$ Medical Faculty, Comenius University, Bratislava, Slovak Republic

Peer-review: Internal review

Conflict of interest: None to declare

Authorship: L.B.

\section{References}

1. Teunissen PW, Dornan T. Lifelong learning at work. BMJ 2008; 336: 667.

2. Siddiqui ZS. Lifelong learning in medical education: from CME to CPD. J Coll Physicians Surg Pak 2003; 13: 44-7.

3. Brandt K. From residency to lifelong learning. J Craniofac Surg 2015; 26: 2287- 8.

4. Hautz SC, Hautz WE, Feufel MA, Spies CD. What makes a doctor a scholar: a systematic review and content analysis of outcome frameworks. BMC Med Educ 2016; 16: 119.

5. Fincher RM, Simpson DE, Mennin SP, Rosenfeld GC, Rothman A, McGrew MC, et al. Scholarship in teaching: an imperative for the 21st century. Acad Med 2000; 75: 887- 94.

6. Collins J. Education techniques for lifelong learning: Lifelong learning in the 21 st century and beyond. Radiographics 2009; 29: 613-22.
7. Glassick CE. Boyer's expanded definitions of scholarship, the standards for assessing scholarship, and the elusiveness of the scholarship of teaching. Acad Med 2000; 75: 877-80.

8. Wagner G, Bacharova L, Timuralp B, Kudaiberdieva G, Uzun C, Inan M, et al. Statement from the International Scientific Summer School Collaboration Group. J Electrocardiol 2014; 47: 126-7.

9. Bacharova L, Kudaiberdieva G, Misak A, Hakacova N, Timuralp B, Wagner GS. The effect of International Scientific Summer School research training on scientific productivity of trainees. Int J Cardiol 2014; 176: 1142-6.

10. Wagner G, Kudaiberdieva G, Bacharova L: International Research Interdisciplinary School (IRIS) initiative sponsorship of journals in training of participants in peer-review. J Electrocardiol 2016; 49: 263-4. 